\section{Regards sur l'économie allemande}

Bulletin économique du CIRAC

$95 \mid 2010$

Varia

\title{
Localisations industrielles : les entreprises redécouvrent les vertus du made in Germany
}

\section{Steffen Kinkel et Spomenka Maloca}

Traducteur : Isabelle Bourgeois

\section{OpenEdition \\ Journals}

Édition électronique

URL : http://journals.openedition.org/rea/4017

DOI : $10.4000 /$ rea. 4017

ISBN : 978-2-8218-0886-7

ISSN : 1965-0787

Éditeur

CIRAC

\section{Édition imprimée}

Date de publication : 1 mars 2010

Pagination : 5-14

ISSN : 1156-8992

\section{Référence électronique}

Steffen Kinkel et Spomenka Maloca, «Localisations industrielles : les entreprises redécouvrent les vertus du made in Germany ", Regards sur l'économie allemande [En ligne], 95 | mars 2010, mis en ligne le 01 mars 2012, consulté le 19 avril 2019. URL : http://journals.openedition.org/rea/4017 ; DOI : $10.4000 /$ rea. 4017 


\section{Localisations industrielles : les entreprises redécouvrent les vertus du made in Germany}

\author{
Steffen Kinkel et Spomenka Maloca
}

L'industrie allemande a subi de plein fouet les répercussions de la crise financière et économique globale. Fortement dépendante du marché mondial, l'économie allemande a vu en 2009 son PIB chuter de $5 \%$ par rapport à 2008. Certaines branches clefs, traditionnellement tournées vers l'export comme la construction mécanique, ont vu leurs entrées de commandes reculer de près de $40 \%$, et leur production baisser de près de $25 \%$. Dès lors se pose la question de savoir quel a été l'impact de la crise sur la tendance des entreprises industrielles à délocaliser leur production. Jusqu'ici en effet, en situation de contexte économique tendu, on a toujours observé un regain des délocalisations en réponse à une pression concurrentielle accrue. Qu'en est-il aujourd'hui ? Les entreprises, voyant baisser le volume de leur production, y ont-elles recouru de manière accrue pour mieux contenir leurs coûts ? Ou ont-elles au contraire plutôt cherché à préserver les capacités existantes en les concentrant sur le marché domestique? L'enquête que nous menons auprès des entreprises industrielles révèle d'importants changements dans leurs activités de délocalisation/ relocalisation : leurs conséquences sont loin d'être négligeables pour la politique industrielle comme la politique de compétitivité du site Allemagne.

\section{Mieux comprendre les stratégies poursuivies par les entreprises dans la crise actuelle}

Ces dernières années, le thème de l'internationalisation des activités est (re)devenu un thème central des préoccupations en matière de politique économique comme des débats sur la stratégie des entreprises, portant principalement sur les raisons qui incitent ces dernières à délocaliser leur production ou le potentiel de compétitivité qu'elles en attendent. Si par le passé, l'implantation de sites de production à l'étranger était pour l'essentiel le fait des grandes multinationales (Dunning, 1980), désormais, un nombre croissant de PME est en quête de nouveaux débouchés à l'étranger ou cherche à mettre à profit les avantages d'une intensification de la division internationale du travail dans la production de biens (Bassen et al., 2001 ; Kinkel/Lay, 2004). Plus particulièrement, les entreprises délocalisent une partie de leur production pour implanter des sites à l'international ou développer ceux qui y existent déjà (DIHK, 2003, 2005 ; Kinkel et al., 2009).

Par délocalisation de la production à l'étranger : offshoring de capacités de production, on entend aussi bien le transfert d'une partie de la chaîne de création de valeur vers les filiales sises à l'étranger (captive offshoring) que vers des fournisseurs ou prestataires de services établis à l'étranger (offshore outsourcing). L'élargissement de l'UE qui accueille désormais douze nouveaux Etats membres a apporté une nouvelle dynamique à ce phénomène (Mattes/Strotmann, 2005).

A la fin des années 1990 et au début du XXI ${ }^{e}$ siècle, les entreprises industrielles allemandes avaient ainsi très largement délocalisé leurs capacités de production (DIHK 2005 ; Kinkel et al. 2009 ; Mattes/Strotmann 2005). Leur objectif était généralement d'améliorer leur positionnement en matière de coûts, notamment dans les nouveaux Etats membres l'UE, mais aussi, dans une moindre mesure,
Steffen Kinkel, économiste et docteur en sciences de l'ingénieur, dirige le Competence Center Industrieund Serviceinnovationen $d u$ Fraunhofer-Institut für Systemund Innovationsforschung (ISI) de Karlsruhe.

Spomenka Maloca, sociologue, y est en charge notamment des enquêtes sur l'innovation.

Un nombre croissant de PME qui délocalisent

Une dynamique accélérée par l'élargissement de l'UE

La délocalisation a longtemps été une stratégie de compression des coûts 
Mais souvent, les risques ont été mal estimés

Une meilleure connaissance des choix des entreprises...

... est indispensable pour clarifier les débats

Quels sont-ils en période de crise? d'accroître leurs performances dans la pénétration du marché local. II n'était alors pas rare non plus qu'elles optent ensuite pour la relocalisation de leurs activités. Elles avaient en effet souvent sous-estimé les risques et les difficultés liés à l'installation de sites de production dans les pays à bas salaires et s'étaient vues contraintes de mettre en place des stratégies d'adaptation à moyen terme (Kinkel et al., 2009). Malgré ces difficultés, la délocalisation de la production a longtemps fait partie du répertoire standard des mesures de réduction des coûts.

Pour les entreprises, le choix d'un site en vue de l'internationalisation de la production revêt une dimension stratégique de la plus haute importance du fait de ses répercussions à long terme sur leur compétitivité (Dunning, 1980 ; Ferdows, 1997). Elles prennent alors leurs décisions selon les bénéfices potentiels que leur offre la conquête de nouveaux marchés pour développer et préserver l'emploi, y compris sur le territoire allemand. Or les risques inhérents aux stratégies d'internationalisation de la production sont évidents: souvent, il y a un monde entre l'approche théorique et sa mise en œuvre dans la pratique ; les entreprises ne sont pas toujours à la hauteur de l'importance stratégique des choix réalisés en matière de localisation ni en ce qui concerne l'appréciation à leur juste valeur des performances de leurs sites d'implantation (Kinkel, 2004). II a ainsi pu être démontré à plusieurs reprises que les décisions de délocalisation motivées par les coûts sont souvent prises dans la précipitation et reposent sur des bases fragiles (Kinkel, 2004 ; van Eenennaam/Brouthers, 1996). Dans certains cas, ce constat débouche alors sur une relocalisation qui pèse lourdement sur l'activité au détriment des salariés (Schulte, 2002). Au total, les délocalisations justifiées par les coûts ne permettent que trop rarement d'améliorer la position concurrentielle de l'entreprise conformément à la stratégie initiale (Kinkel/Lay 2004).

L'analyse empirique des décisions menant à une délocalisation ou à une relocalisation, et surtout des raisons qui les motivent, s'impose donc tout particulièrement puisqu'elle permet de mettre en évidence les facteurs dont une entreprise devra mieux tenir compte avant d'effectuer ses choix de localisation. Les facteurs de risque ainsi révélés pourront alors être intégrés d'une manière appropriée dans les modèles d'évaluation des performances et perspectives de l'entreprise, mais aussi mieux permettre de thématiser chances et risques du processus de délocalisation de la production dans le débat sur la politique économique.

L'internationalisation de la production est aussi un enjeu fondamental pour la compétitivité d'un pays, avec un impact indéniable sur les marchés de l'emploi tant régionaux que nationaux (Porter, 1990, 1999). La recherche en sciences économiques a, d'un côté, mis en évidence une série d'éléments permettant de conclure que cette internationalisation peut, à long terme, présenter des avantages aussi bien pour les pays d'origine que ceux de destination (Barba/ Navaretti/Falzoni, 2004). D'un autre côté, elle a révélé une série d'indices indiquant que ce sont surtout les délocalisations mues par les coûts qui ont tendance à se substituer aux capacités de production comme aux exportations domestiques, ce qui peut avoir un impact désastreux sur le marché de l'emploi du pays d'origine (Ahlers et al., 2007 ; Römer, 2007 ; Mucchielli/Saucier, 1997).

L'évaluation des délocalisations de la production dans l'industrie allemande, réalisée à la mi-2006 dans le cadre de l'enquête de l'Institut Fraunhofer ISI de Karlsruhe, avait révélé un recul de cette tendance. Dans la phase de croissance économique de l'époque, les entreprises étaient plus nombreuses à investir en vue d'une expansion qu'à délocaliser pour des raisons de coûts. Or par le passé, les délocalisations avaient toujours tendance à s'intensifier en période économique difficile en raison d'une pression concurrentielle accrue. Dans le contexte de la crise économique actuelle se pose dès lors la question de savoir dans quelle mesure l'industrie allemande a modifié ses stratégies de délocalisation. Les entreprises y ont-elles recouru plus massivement afin de mieux maîtriser leurs coûts au regard d'un volume de production en recul ? Ou ont-elles plutôt essayé de préserver leurs capacités de production en concentrant leurs sites allemands? Ces questions trouvent des éléments de réponse scientifiquement fondés dans 
l'édition 2009 de l'enquête « Modernisation de la production » menée par l'Institut Fraunhofer ISI (Karlsruhe) tous les deux ans depuis 1993; depuis 2003, elle fait partie intégrante du European Manufacturing Survey (EMS).

\section{L'enquête « Modernisation de la production » de l'Institut Fraunhofer ISI}

Cette base de données couvre l'ensemble des branches de la production manufacturière dans le but d'observer de manière systématique le degré de modernité et de compétitivité des structures de production. L'étude a pour objet les stratégies de production poursuivies, la mise en œuvre de concepts organisationnels et techniques innovants dans la production, l'affectation des ressources humaines, la gestion des qualifications, ainsi que les questions relatives au choix des sites de production. L'enquête porte en outre sur des indicateurs de performance tels que la productivité, la flexibilité et la qualité du secteur manufacturier allemand, et elle offre un regard actuel sur la réalité des projets de délocalisation des entreprises industrielles. Ce sondage est le seul à éclairer de manière détaillée la problématique des relocalisations et à en tirer les enseignements en ce qui concerne les sources d'erreur constatées dans les stratégies de délocalisation, de même que certains avantages sous-estimés du site industriel allemand. Réalisé pour le compte de l'Association des ingénieurs allemands (Verein Deutscher Ingenieure, VDI), ce sondage répond ainsi aux questions clefs suivantes:

- Combien d'entreprises industrielles, et lesquelles, ont délocalisé/ relocalisé une partie de leur production entre 2007 et la mi-2009 ? - Combien d'entreprises projettent actuellement de délocaliser leur production ou au contraire de la relocaliser pour mieux exploiter leurs capacités en Allemagne?

- Quels sont les destinations privilégiées et à partir de quels pays s'effectuent les relocalisations ?

- Quels sont les critères déterminants pour les délocalisations et les relocalisations?

- Quelle importance revêt l'externalisation de capacités de production auprès de fournisseurs et prestataires étrangers (offshore outsourcing) dans le cadre des délocalisations de la production?

La présente analyse repose sur les données de la vague d'enquête de 2009, au cours de laquelle 15576 entreprises industrielles sises en Allemagne ont été contactées par écrit. En août 2009, 1484 entreprises avaient retourné un questionnaire complété et exploitable (taux de réponse : $10 \%$ ). Les entreprises ayant répondu recouvrent la totalité des secteurs industriels. La construction mécanique et l'industrie des métaux sont représentées respectivement à hauteur de $19 \%$ et $17 \%$, l'industrie électrotechnique à $15 \%$, l'industrie du papier, de l'édition et de l'impression à $5 \%$, et l'industrie agroalimentaire à $8 \%$. Les entreprises de moins de 100 salariés constituent $63 \%$ des répondants, les entreprises de taille moyenne $33 \%$, et les grandes entreprises (plus de 1000 salariés) $4 \%$. L'exploitation des réponses a été pondérée en fonction de la structure par branche, par taille et par régions de l'ensemble du secteur manufacturier afin d'optimiser la représentativité de la base de données.

\section{La crise incite plutôt à préserver les capacités existantes}

L'analyse sur la durée de l'industrie révèle clairement que la délocalisation de la Délocalisations en recul production est actuellement en recul. Seulement $9 \%$ des entreprises industrielles allemandes ont délocalisé une partie de leur production à l'étranger entre 2007 et la mi-2009, c'est-à-dire sur une période comprenant environ une année de pleine exploitation des capacités et près d'une année de crise. Rapportée au taux déjà faible (15\%) enregistré lors de l'enquête précédente menée entre la mi-2004 et la mi-2006, la part des entreprises qui délocalisent s'est nettement réduite. Les branches de la métallurgie et de l'électrotechnique affichent leur plus bas taux de délocalisation depuis le milieu des années 1990. On constate ainsi pour la première fois une baisse des délocalisations en période de marasme économique.

Evolution des délocalisations et relocalisations depuis 1995

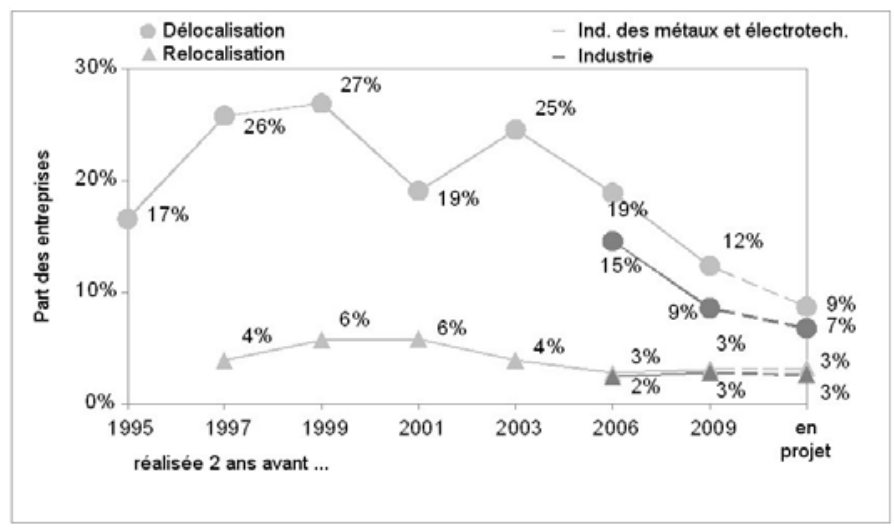

Source : Fraunhofer ISI, sondages Modernisierung der Produktion, données pondérées.

Visiblement, la crise actuelle incite les entreprises à préférer maintenir leurs capacités sur les sites de production existants plutôt que de se mettre en quête de 
Bientôt une entreprise qui relocalise pour deux qui délocalisent

Plus les entreprises sont grandes plus elles délocalisent

$3 \%$ des entreprises industrielles relocalisent, surtout les PME

Electrotechnique, automobile et textile-habillement en tête pour la délocalisation

Recul des délocalisations notamment dans l'automobile et la construction mécanique possibilités supplémentaires pour réduire leurs coûts par le transfert d'activités dans les pays à bas salaires. Les données relatives aux délocalisations actuellement en projet ( $7 \%$ des entreprises) confirment la réserve actuelle de l'industrie.

Dans le même temps, l'évolution de la relocalisation de capacités de production auparavant délocalisées est restée stable : avec environ $3 \%$, la part des entreprises qui rapatrient ces capacités est restée à peu près constante depuis 2003. Actuellement, on compte donc une entreprise qui relocalise pour trois qui délocalisent. Les projets de relocalisation formulés pour optimiser l'utilisation des capacités de production domestique laissent présumer eux aussi une stabilisation. Et on peut estimer que, dans les deux ou trois années à venir, sur deux entreprises qui délocalisent, il y en aura une qui rapatriera ses capacités.

L'intensité des délocalisations varie de manière significative selon la taille des entreprises. Alors que $45 \%$ des grandes entreprises (plus de 1000 salariés) délocalisent, ce taux se réduit au fil des autres catégories pour atteindre $24 \%, 10 \%$ et jusqu'à $3 \%$ seulement pour les entreprises de moins de 50 salariés. Du fait que les PME représentent la majeure partie des entreprises, une projection du nombre absolu par an montre que, sur le total des 1750 entreprises qui délocalisent, près de la moitié (840) emploie de 50 à 249 salariés. Vient ensuite le groupe des grosses PME (de 250 à 999 salariés) avec près de 390 entreprises qui délocalisent, suivi des petites (330) et grandes entreprises (190).

Délocalisations et relocalisations selon la taille de l'entreprise

\begin{tabular}{|l|cc|cc|}
\hline & \multicolumn{2}{|c|}{ Délocalisations (par an *) } & \multicolumn{2}{c|}{ Relocalisations (par an *) } \\
& $\begin{array}{l}\text { Nombre d'entr. } \\
\text { Moins de } \mathbf{5 0} \text { salariés }\end{array}$ & 330 & $3 \%$ & \multicolumn{2}{c|}{ Nombre d'entr. } & en \% \\
$\mathbf{5 0 - 2 4 9}$ salariés & 840 & $10 \%$ & 160 & $2 \%$ \\
$\mathbf{2 5 0}-\mathbf{9 9 9}$ salariés & 390 & $24 \%$ & 120 & $3 \%$ \\
$\mathbf{1 0 0 0}$ salariés et plus & 190 & $45 \%$ & 50 & $7 \%$ \\
Ensemble de l'industrie & 1750 & $9 \%$ & 570 & $3 \%$ \\
\hline
\end{tabular}

Source : Fraunhofer ISI, sondages Modernisierung der Produktion, données pondérées. *) Projection sur l'ensemble du secteur manufacturier.

La répartition des relocalisations en Allemagne selon la taille des entreprises présente un profil similaire. Entre 2007 et la mi-2009, près de $3 \%$ des entreprises ont procédé à des relocalisations, soit environ 570 entreprises par an. Dans ce cas aussi, l'intensité des relocalisations va decrescendo selon la taille de l'entreprise, passant de $11 \%$ à moins de $2 \%$. En valeur absolue, le groupe le plus actif est celui des PME de 50 à 249 salariés, avec 240 entreprises concernées. Ces données prouvent que les relocalisations, loin de ne concerner que quelques cas isolés, sont au contraire un phénomène parfaitement mesurable.

L'analyse par branches fait apparaître des spécificités sectorielles. Les entreprises qui recourent le plus souvent aux délocalisations sont celles de l'électrotechnique (23\%), de la construction et de l'équipement automobile (20\%), ainsi que de l'industrie du textile-habillement (16\%). L'éventail des branches largement et moyennement impliquées s'étend de la construction mécanique à la production de caoutchouc et de matières plastiques, en passant par la production d'appareils de mesure et d'asservissement ou de l'industrie optique et médicale, la chimie ou le travail des métaux. Plus fortement tributaires de leur clientèle locale, les secteurs de l'agroalimentaire ou de l'industrie du papier et de l'impression ne délocalisent en revanche que très peu.

En comparaison avec l'intensité des délocalisations sur la période d'observation de mi-2004 à mi-2006, des reculs très nets (d'environ $10 \%$ dans chaque branche) s'observent dans la construction automobile et mécanique, dans la production de caoutchouc et de matières plastiques, ainsi que dans le textilehabillement. Chez les constructeurs et équipementiers automobiles comme dans la construction mécanique, c'est tout particulièrement la baisse significative des entrées de commandes comme des ventes qui a à l'évidence freiné les délocalisations. Dans le secteur de l'industrie électrotechnique, on observe en revanche 
une baisse relativement faible de la propension à délocaliser, à partir d'un niveau assez élevé, il est vrai. Dans cette branche, visiblement, malgré une baisse prévisionnelle du chiffre d'affaires, la concurrence mondiale et la pression sur les coûts sont si fortes que les entreprises continuent à épuiser toutes les possibilités de rationaliser les coûts grâce à la production dans les pays à bas salaires.

Délocalisations et relocalisations par branche (part des entreprises)

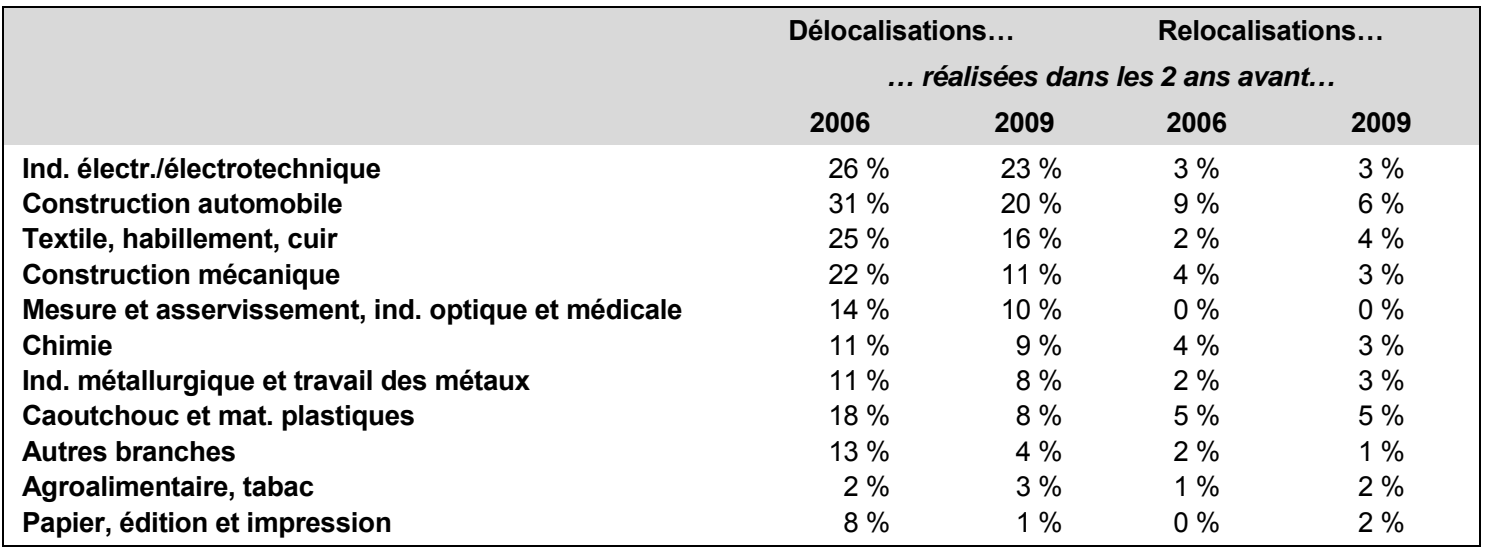

Source : Fraunhofer ISI, sondages Modernisierung der Produktion, données pondérées.

En ce qui concerne les activités de relocalisation, les différences entre les branches sont moins prononcées que lors de la période d'observation précédente. Un recul significatif (3\%) ne s'observe que dans la construction et l'équipement automobile: la part des entreprises qui rapatrient leurs activités y est tombée à $6 \%$. Malgré cette baisse, c'est toujours la construction automobile qui enregistre le taux de relocalisation le plus élevé, suivie de près par la production de caoutchouc et de matières plastiques. Pour des raisons structurelles, en effet, l'industrie automobile cherche encore à épuiser toutes les possibilités pour concentrer ses capacités de production.

Délocalisation selon les priorités stratégiques des entreprises

\begin{tabular}{|lc|}
\hline & En \% des entreprises \\
Prix des produits & $14 \%$ \\
Produits innovants & $12 \%$ \\
Délais de livraison/respect des engagements & $8 \%$ \\
Adaptation des produits à la demande du client & $7 \%$ \\
Qualité des produits & $7 \%$ \\
\hline
\end{tabular}

Source : Fraunhofer ISI, sondages Modernisierung der Produktion, données pondérées.

Quand on interroge les entreprises sur leur priorité stratégique pour aborder la concurrence, on constate également des différences dans leur propension à délocaliser. Les entreprises où cette propension est la plus élevée sont celles qui misent d'abord sur la compétitivité-prix : elles représentent $14 \%$ du total. Cela n'a rien d'étonnant, puisque ces mêmes entreprises cherchent plus que les autres à comprimer les coûts salariaux en délocalisant dans les pays à bas salaires ; l'objectif étant d'optimiser le facteur clef sur lequel repose leur compétitivité.

Délocalisent également plus que la moyenne les entreprises qui se positionnent comme leader en matière d'innovation. Elles mènent une double stratégie, concentrant sur le marché domestique les activités de développement et de production de produits innovants, et cherchant en même temps à préserver leur compétitivité-prix grâce aux délocalisations. Ce constat amène à réviser l'hypothèse commune selon laquelle plus une entreprise est innovante, moins elle serait encline à délocaliser.

A l'opposé, les entreprises qui sont leaders en matière de qualité et de réactivité, c'est-à-dire qui se positionnent sur l'adaptation permanente de leurs produits aux attentes des clients, sont celles qui délocalisent le plus rarement leur production à
Relocalisations : automobile en têteL'automobile toujours en tête

pour les relocalisations

La tendance à délocaliser est forte chez les entreprises misant sur la compétitivité-prix,...

... supérieure à la moyenne chez celles misant sur l'innovation,...

... et rare chez celles misant sur la qualité 
Les entreprises délocalisent pour comprimer les coûts salariaux, se rapprocher de leurs débouchés... l'étranger. Dans cette catégorie, la crainte de perdre les facteurs clefs sur lesquels repose la compétitivité de l'entreprise, à savoir la qualité des produits et la réactivité à la demande (voir les motifs de relocalisation), trouve semble-t-il son reflet dans une stratégie avant tout axée sur la réputation du made in Germany, et qui s'accommode donc mal de délocalisations.

\section{Le souci de la qualité l'emporte sur la compression des coûts}

La compression des coûts salariaux reste la principale raison incitant à délocaliser: elle est invoquée par les trois quarts des entreprises qui délocalisent. Viennent ensuite au deuxième et troisième rang des motifs, avec respectivement près de $30 \%$ des réponses, la proximité du site de production avec les clients stratégiques implantés à l'étranger et la conquête de nouveaux marchés dans le pays de destination. Le critère de la proximité avec ces clients clefs a gagné en importance depuis l'enquête menée à la mi-2006: le taux de réponses a augmenté de 8 points de pourcentage. II convient ici de rappeler que ce critère avancé ne reflète pas seulement la stratégie proactive des entreprises, mais traduit aussi le fait que les clients stratégiques demandent souvent expressément à leurs fournisseurs de venir produire à proximité de leurs sites étrangers.

... et pour réduire les coûts de transport

L'impératif de proximité avec des capacités de production déjà délocalisées, ainsi que la quête d'une réduction des coûts de transport et de logistique en localisant la production à proximité de la demande représentent, quasi ex aequo avec environ $15 \%$ des citations, les quatrième et cinquième motifs de délocalisation. La question des coûts de transport est déterminante, surtout pour une entreprise qui choisit de livrer directement les clients du pays visé ou des marchés-passerelle depuis le lieu de production délocalisé, et décide au contraire de ne pas le faire via la voie détournée qu'est la réimportation en Allemagne de la production confiée à son 'atelier prolongé' à l'étranger. Dans ce cas aussi, la desserte du marché local fait donc partie intégrante de la stratégie de production à l'étranger. La quête d'un environnement plus porteur en matière d'impôts, de taxes et de subventions n'est, comme en 2006, un critère prioritaire que pour moins de $10 \%$ des entreprises qui délocalisent. Quant au manque de main-d'œuvre qualifiée, il n'a qu'un très faible impact: le déficit de main-d'œuvre qui se creuse en Allemagne dans certaines filières ne semble pas encore jouer de rôle notable sur la décision de délocaliser des capacités de production.

Motifs de délocalisation et de relocalisation

\begin{tabular}{|lc|}
\hline & En \% des entreprises \\
Coûts salariaux & $77 \%$ \\
Proximité avec les clients stratégiques & $29 \%$ \\
Conquête de nouveaux marchés & $28 \%$ \\
Proximité avec des sites de production déjà délocalisés & $16 \%$ \\
Coût de transport, de logistique & $15 \%$ \\
Impôts, taxes, subventions & $12 \%$ \\
Manque de main-d'œuvre qualifiée & $8 \%$ \\
Accès aux nouveaux savoirs/technologies/clusters & $2 \%$ \\
\hline
\end{tabular}

Source : Fraunhofer ISI, sondages Modernisierung der Produktion, données pondérées.

Elles relocalisen parce qu'elles ont rencontré des problèmes de qualité,...

L'analyse des principaux motifs de relocalisation permet de déceler les facteurs qui n'ont pas été pris suffisamment en considération par les entreprises quand elles ont opté pour une délocalisation ; elle révèle aussi les cas où les entreprises se sont vues contraintes d'adapter leur stratégie face à la dynamique évolutive de l'environnement de leur site délocalisé. La principale raison qui les incite à relocaliser leur production est qu'elles ont rencontré des problèmes de qualité. Ils résultent d'une sous-estimation du laps de temps nécessaire pour garantir, dans des pays de langue et de culture différentes, la qualité souhaitée des produits comme des process de production et, par répercussion, d'une sous-estimation des coûts accompagnant en interne le contrôle qualité. Ce motif a continué de gagner en importance depuis l'enquête de la mi-2006. 
Le deuxième motif de relocalisation, invoqué par 4 entreprises sur 10 , est une baisse critique de leur réactivité comme de leur capacité à livrer le client dans les délais. Elle peut aussi bien provenir de problèmes survenus dans la production propre de l'entreprise que de problèmes rencontrés lors du recours à des réseaux de fournisseurs locaux mobilisés ad hoc. On comprend dès lors mieux aussi pourquoi ce sont justement les entreprises dont la stratégie de compétitivité est axée sur la réactivité dans l'ajustement de leur production et dans la réponse à la demande du client qui sont les plus réticentes à envisager une délocalisation.

Les motifs poussant à la relocalisation

\begin{tabular}{|lc|}
\hline & En \% des entreprises \\
Qualité & $68 \%$ \\
Réactivité, flexibilité, capacité à livrer le client & $43 \%$ \\
Coûts salariaux & $33 \%$ \\
Coûts de transport, logistique & $32 \%$ \\
Dépenses de coordination, de contrôle & $20 \%$ \\
Disponibilité, fluctuation de la main-d'œuvre qualifiée & $19 \%$ \\
Perte de savoir-faire, copie, contrefaçon & $5 \%$ \\
Proximité de la R\&D sur le marché domestique & $2 \%$ \\
\hline
\end{tabular}

Source : Fraunhofer ISI, sondages Modernisierung der Produktion, données pondérées.

En troisième et quatrième position sont invoqués, avec respectivement un tiers des citations, les coûts salariaux à l'étranger ainsi que ceux liés au transport et à la logistique. L'importance accordée aux coûts salariaux a fortement progressé depuis l'enquête réalisée à la mi-2006 (le taux de citation a plus que doublé), ce qui traduit une dynamique de hausse des salaires d'une ampleur inattendue dans les pays de destination. Comme beaucoup d'entreprises avaient effectué une lecture statique des avantages comparés des divers sites ou sous-estimé l'ampleur des évolutions, nombre des avantages escomptés à l'origine d'une délocalisation se sont depuis mués en leur contraire. L'invocation de la sous-estimation des coûts de transport et de logistique révèle que, surtout dans le cas d'une stratégie misant sur l'implantation à l'étranger d'un 'atelier prolongé' dont la production est ensuite réimportée en Allemagne, ce facteur coûts peut rapidement prendre des dimensions réduisant à néant les avantages initialement attendus du différentiel salarial. S'y ajoute que, pour une entreprise sur cinq, la sous-estimation des coûts engendrés par la coordination et le contrôle qualité, depuis le siège, du site de production à l'étranger représente un motif essentiel de relocalisation.

Quant à la valeur accordée à la disponibilité ou à la fluctuation de la maind'œuvre qualifiée, elle a elle aussi doublé depuis la dernière enquête. Ainsi, ce facteur détermine plus souvent les entreprises à délocaliser que le manque de main-d'œuvre qualifiée en Allemagne ne les incite à délocaliser. En revanche, les risques liés à une perte du savoir-faire ou à la contrefaçon ne jouent encore qu'un rôle secondaire. Cela vaut également pour la proximité de la R\&D sur le marché domestique. Dans les deux cas, cette faible importance s'explique par le fait que par le passé, c'est plutôt la production de produits matures, situés à un stade avancé de leur cycle de vie, qui avait été délocalisée (Kinkel et al. 2009).

\section{Europe de l'est : attractivité en baisse ; Asie : attractivité en hausse}

Etant donné l'importance accordée au facteur coût, les nouveaux Etats membres de l'UE sont la destination la plus prisée : $40 \%$ des entreprises y ont délocalisé leur production entre 2007 et la mi-2009. Toutefois, l'attractivité de ces Etats a considérablement reculé. Lors de l'enquête menée à la mi-2006, le taux des délocalisations dans ces pays atteignait encore 15 points de plus. Aujourd'hui, la moitié des relocalisations s'effectue à partir des nouveaux Etats membres de l'UE. Cette proportion, qui a augmenté de 12 points par rapport à l'enquête précédente, confirme elle aussi l'attractivité déclinante de ces pays d'Europe centrale
... qu'elles voient leur réactivité réduite...

... ou qu'elle affrontent une explosion des coûts

Le critère de la disponibilité d'une main-d'œuvre qualifiée gagne en importance

Recul de l'attractivité des nouveaux Etats membres de l'UE 
et orientale comme site de production pour les entreprises allemandes. Parallèlement, les délocalisations vers les autres Etats est-européens ont tendance à stagner elles aussi, tandis que les relocalisations depuis ces pays, qui n'étaient pas mesurables en 2006, prennent une importance croissante (9\%). Etant donné l'importance des coûts salariaux pour les choix de relocalisation, l'une des raisons expliquant la perte d'attractivité de ces territoires est vraisemblablement la hausse, parfois conséquente, des salaires observée dans certaines régions de la Pologne, de la République Tchèque, de la Hongrie ou de la Slovaquie.

Origine et destination des délocalisations et relocalisations

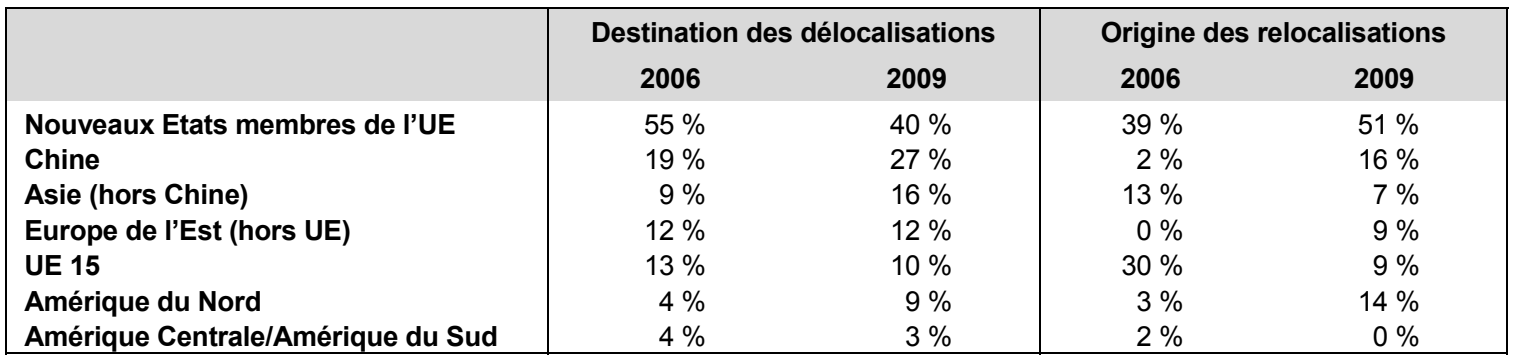

Source : Fraunhofer ISI, sondages Modernisierung der Produktion, données pondérées.

L'attractivité de la Chine en hausse mais les relocalisations augmentent

L’Asie, région privilégiée pour les bas salaires

La crise incite à réduire l'activité en Amérique du Nord mais pas dans l'UE 15

a crise incite les entreprises à accroître le taux d'utilisation de leurs capacités..
La deuxième destination est la Chine, où $27 \%$ des entreprises ont délocalisé leur production; ce taux a augmenté de 8 points depuis la précédente enquête. Fait révélateur : la Chine n'est plus considérée comme une destination attractive par les seules grandes entreprises, mais aussi, dans une proportion aujourd'hui comparable, par les PME. D'un autre côté, le taux de relocalisation en provenance de Chine s'élève désormais à $16 \%$; pour la première fois, cette tendance est mesurable. Elle concerne surtout les petites et moyennes entreprises qui, en raison de leur plus grand éloignement à la fois physique et culturel, ont plus de difficultés à résoudre les problèmes récurrents qui se posent en termes de qualité.

Les autres pays asiatiques ont eux aussi considérablement gagné en attractivité se situant désormais, avec $16 \%$ des entreprises qui délocalisent (5 points de plus que lors de l'enquête précédente), au troisième rang des destinations. Au regard à la fois de l'importance croissante de la Chine comme de la perte d'attractivité des nouveaux Etats de l'UE, on peut en conclure que les entreprises qui délocalisent pour comprimer leur masse salariale préfèrent aujourd'hui d'entrée de jeu s'installer dans les pays asiatiques certes plus éloignés, mais aux coûts salariaux inférieurs, que dans les nouveaux Etats membres géographiquement plus proches, mais où les niveaux des salaires ont connu une nette hausse.

Les pays de l'UE 15 (d'avant l'élargissement) et l'Amérique du Nord viennent en cinquième et sixième position. Cette dernière destination a vu son attractivité augmenter de 5 points depuis la dernière enquête, la dépréciation du dollar intervenue depuis ayant ouvert des perspectives de développement à nombre d'entreprises allemandes pour leurs stratégies de production ou d'approvisionnement. Or parallèlement, les relocalisations en provenance d'Amérique du Nord ont connu elles aussi une nette hausse $(11 \%)$ et s'élèvent désormais à $14 \%$. La raison en est vraisemblablement la crise économique mondiale et la récession qui a touché très tôt le marché américain que nombre d'entreprises industrielles allemandes considéraient jusque là comme leur principal débouché. Quant aux relocalisations en provenance des 15 Etats de l'UE d'avant son élargissement, leur niveau $(9 \%)$ n'est plus guère significatif aujourd'hui, alors que jusqu'en 2003 cette région était la première d'où les entreprises rapatriaient leurs installations.

\section{Captive offshoring : mode de délocalisation privilégié}

Les activités de délocalisation se scindent en deux catégories principales : la délocalisation de capacités de production au sein de filiales à l'étranger (captive offshoring) et l'externalisation de la production auprès d'entreprises sous-traitantes 
étrangères (offshore outsourcing). Sur la période de 2007 à la mi-2009, plus de $60 \%$ des entreprises qui délocalisent ont eu recours au captive offshoring. Près d'un tiers d'entre elles ont préféré l'offshore outsourcing, tandis que $4 \%$ ont opté pour les deux stratégies. Si on compare ce profil avec celui de l'enquête de 2006, on s'aperçoit que le recours au captive offshoring a augmenté d'environ 10 points alors que la pratique du offshore outsourcing a diminué. Cette tendance révèle le comportement des entreprises en temps de crise : elles préfèrent accroître le taux d'utilisation de leurs propres capacités de production, y compris à l'étranger, ce qui les amène souvent à réduire le transfert de capacités de production à leurs sous-traitants externes.

Evolution des modes de délocalisation de la production

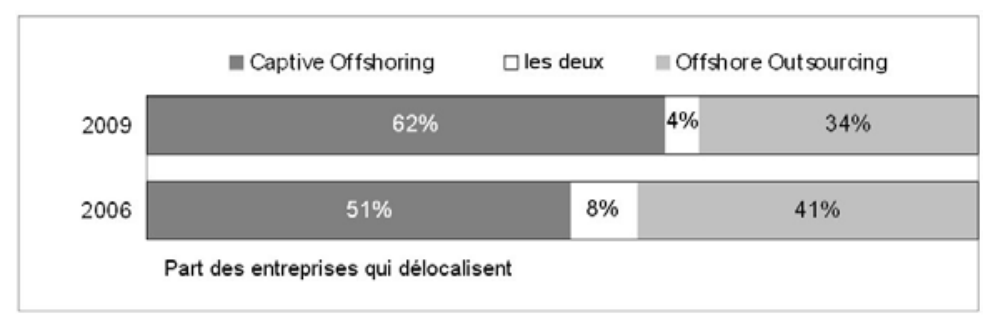

Source : Fraunhofer ISI, sondages Modernisierung der Produktion, données pondérées.

D'autres analyses, plus détaillées, révèlent que ce sont plutôt les grandes entreprises qui optent pour le captive offshoring, alors que les PME misent davantage sur l'offshore outsourcing. On observe aussi des différences significatives quant aux critères qui motivent ces choix. Dans le cas du captive offshoring, ce sont la conquête de nouveaux marchés dans le pays de destination ou encore la proximité avec le client stratégique à l'étranger qui l'emportent en tendance. A l'inverse, c'est plutôt l'objectif de réduction des coûts qui détermine une entreprise à opter pour l'offshore outsourcing. Quant au glissement observé vers les stratégies " captives ", il témoigne de la tendance qu'ont actuellement les entreprises à privilégier les stratégies proactives, axées sur le client et la conquête d'un marché ; elles délocalisent moins pour réduire leurs coûts.

LA CRISE A MODIFIÉ SIGNIFICATIVEMENT L'APPROCHE DES ENTREPRISES industrielles en matière de délocalisations, ainsi que le révèlent nos analyses. Les activités de délocalisation de la production à l'étranger sont tombées à leur plus bas niveau depuis quinze ans. Dans le même temps, le nombre des relocalisations vers l'Allemagne est resté stable. On compte désormais une entreprise qui relocalise pour trois entreprises qui délocalisent. Si les décisions de relocalisation résultent en premier lieu de problèmes de qualité rencontrés à l'étranger, la dynamique salariale, la fluctuation ou le manque de main-d'œuvre qualifiée ont gagné en importance. Au niveau des destinations, on constate un recul de l'attractivité des nouveaux Etats membres de l'UE : les délocalisations vers ces pays ont fortement reculé, les relocalisations considérablement augmenté. A l'opposé, la Chine et les autres régions d'Asie ont encore gagné en attractivité, même si entretemps, on peut observer une tendance désormais mesurable à la relocalisation en provenance de ces régions.

La première conclusion qu'on peut en tirer est positive. Le site industriel allemand reste attractif : dans le contexte de la crise économique actuelle, les entreprises semblent donner priorité à la préservation des capacités existantes en Allemagne. Le fait que, dans certains pays étrangers, les procédures de fermeture d'un site sont plus flexibles, y contribue assurément. La décision se trouve en outre favorisée lorsque, comme aux Etats-Unis ou dans les nouveaux Etats membres est-européens de l'UE, un marché en plein boom avant la crise voit s'effondrer la demande dans des proportions parfois dramatiques et que cela tasse l'utilisation des capacités de production qui y sont installées.

II faut toutefois se garder d'en conclure que délocaliser des capacités de production à l'étranger ne serait plus désormais considéré comme une priorité straté-
... et à renforcer leurs stratégies de fidélisation du client comme de conquête des marchés 
gique ni que la dynamique se trouverait aujourd'hui enrayée, tout ce qui étant 'délocalisable' ayant déjà été transféré à l'étranger. L'équilibre du partage mondial du travail est un processus dynamique où entrent en jeu les potentiels de croissance et d'innovation à l'échelon tant local que global. Les analyses antérieures nous ont appris que, pour des raisons de coûts, les entreprises délocalisent de préférence vers les pays à bas salaires la production de biens matures, requérant des process standardisés. Or les produits et process considérés comme innovants aujourd'hui, au pilotage intensif en savoir-faire, auront valeur de standard d'ici trois à cinq ans, et seront alors 'mûrs' pour être délocalisés et développer ainsi un nouveau potentiel d'efficience. Considéré sous cet angle dynamique, le mouvement des délocalisations de production ne s'arrêtera jamais. Et tout porte à croire que, avec la reprise au sortir de la crise, quand l'utilisation des capacités domestiques sera revenue au maximum, les entreprises allemandes chercheront à nouveau à réaliser à l'étranger leur stratégie de compression des coûts. II reste néanmoins à espérer qu'elles auront alors tiré les enseignements des risques auxquels les ont exposées leurs choix passés de délocaliser trop exclusivement pour des raisons de coûts, sans tenir compte des facteurs qualitatifs essentiels pour leur compétitivité : la qualité des produits et leur réactivité. Car une relocalisation 'revient cher', dans tous les sens du terme.

Traduction : I. Bourgeois et S. Hazouard

\section{Indications bibliographiques}

- Ahlers E., Öz F., Ziegler A., Standortverlagerung in Deutschland - einige empirische und politische Befunde, Düsseldorf, 2007

- Barba Navaretti G., Falzoni, A.M., « Home Country Effects of Foreign Direct Investment », in Barba Navaretti G., Venables A.J. (eds.), Multinational Firms in the World Economy, Princeton University Press, Princeton/Oxford, 2004

- Bassen A., Behnam M., Gilbert D.U., « Internationalisierung des Mittelstands. Ergebnisse einer empirischen Studie zum Internationalisierungsverhalten deutscher mittelständischer Unternehmen ", Zeitschrift für Betriebswirtschaft, $\mathrm{n}^{\circ}$ 4, 2001

- DIHK (ed), Produktionsverlagerung als Element der Globalisierungsstrategie von Unternehmen. Ergebnisse einer Unternehmensbefragung, Deutscher Industrie- und Handelskammertag, Berlin, 2003

- DIHK (ed), Going International. Erfolgsfaktoren im Auslandsgeschäft, Deutscher Industrie- und Handelskammertag, Berlin, 2005

- DuNNING J.H., « Towards an eclectic theory of international production : some empirical tests », in Journal of International Business Studies, $\mathrm{n}^{\circ}$ 1, 1980

- EenennaAm F. VAN, Brouthers K., « Global Relocation : High Hopes and Big Risks », in Long Range Planning, $\mathrm{n}^{\circ} 1,1996$

- FERDOWs K., "Making the most of foreign factories », in Harvard Business Review, n 2, 1997

- KINKEL S. (ed), Erfolgsfaktor Standortplanung. In- und ausländische Standorte richtig bewerten, Berlin, Heidelberg, New York, 2004

- KINKEL S., LAY G., « Motive, strategische Passfähigkeit und Produktivitätseffekte des Aufbaus ausländischer Produktionsstandorte : Ergebnisse einer repräsentativen Erhebung bei 1630 Betrieben des deutschen verarbeitenden Gewerbes », in ZfB Zeitschrift für Betriebswirtschaft, $n^{\circ}$ 5, 2004

- KInkel S., Maloca S., JÄger, A., Produktions- und FuE-Verlagerungen ins Ausland - Verbreitung, Motive und strategische Implikationen für das deutsche verarbeitende Gewerbe, Fraunhofer IRB Verlag, ISI-Schriftenreihe Innovationspotenziale, Karlsruhe, 2009

- Mattes A., Strotmann H., Ausmaß und Bestimmungsgründe des Offshoring in die mittel- und osteuropäischen Beitrittsländer der EU, IAW-Kurzbericht 5/2005, Tübingen

- Mucchielli J.-L., SAUCier P., « European industrial relocations in low-wage countries : policy and theory debates », in BuCKLEY P.J., MucCHIELLI J.-L. (eds.), Multinational Firms and International Relocation, Cheltenham/UK, 1997

- Porter M. E., The Competitive Advantage of Nations, Harvard Business School, London, 1990

- Römer C., Offshoring - Wie viele Jobs gehen ins Ausland ? Auslandsinvestitionen, Produktionsverlagerungen und Arbeitsplatzeffekte, IW Köln, 2007

- Schulte A., Das Phänomen der Rückverlagerung, Internationale Standortentscheidungen kleiner und mittlerer Unternehmen, Wiesbaden, 2002. 